\title{
CORTICAL VISUAL DYSFUNCTION IN CHILDREN: A CLINICAL STUDY
}

\author{
GORDON DUTTON, JOAN BALLANTYNE, GILLIAN BOYD, MICHAEL BRADNAM, \\ RUTH DAY, DAPHNE McCULLOCH, ROISIN MACKIE, SHEELAGH PHILLIPS and \\ KATHRYN SAUNDERS \\ Glasgow
}

\begin{abstract}
SUMMARY
Damage to the cerebral cortex was responsible for impairment in vision in 90 of 130 consecutive children referred to the Vision Assessment Clinic in Glasgow. Cortical blindness was seen in 16 children. Only 2 were mobile, but both showed evidence of navigational blind-sight. Cortical visual impairment, in which it was possible to estimate visual acuity but generalised severe brain damage precluded estimation of cognitive visual function, was observed in 9 children. Complex disorders of cognitive vision were seen in 20 children. These could be divided into five categories and involved impairment of: (1) recognition, (2) orientation, (3) depth perception, (4) perception of movement and (5) simultaneous perception. These disorders were observed in a variety of combinations. The remaining children showed evidence of reduced visual acuity and/ or visual field loss, but without detectable disorders of cognitive visual function. Early recognition of disorders of cognitive vision is required if active training and remediation are to be implemented.
\end{abstract}

A large proportion of the posterior region of the cerebral cortex is devoted to vision. Focal disruption of brain tissue in this area can result in a wide range of disorders of cognitive visual function. ${ }^{1-3}$ While these disorders have been well described in adults, ${ }^{1-3}$ there have been few reports investigating the range of analogous dysfunction in children.

Severe impairment of vision due to cerebral cortical damage in children comprises a small but significant proportion of blind registrations, ${ }^{4}$ but under-reporting of blindness in children is common. ${ }^{4}$ Children who have many handicaps due to pathology of the cerebral cortex commonly show evidence of a

From: Vision Assessment Team, Royal Hospital for Sick Children, Glasgow, UK.

Correspondence to: Mr G. Dutton, Royal Hospital for Sick Children, Yorkhill, Glasgow G3 8SJ, UK. range of complex visual problems of varying severity which are difficult both to elicit and to classify. Even a measure of visual acuity can sometimes be difficult to obtain on account of intellectual or communication dysfunction. Recourse must, therefore, be made to structured interviews with patients and carers who have had the opportunity to observe the child's behaviour in the long term.

We have recently set up a multi-disciplinary paediatric Vision Assessment Clinic in Glasgow, Scotland, in which the majority of referrals comprise those with cortical visual impairment. Detailed interviews are conducted with parents and/or carers and a range of tests of visual function are carried out. A detailed report is written for parents, teachers and carers concerning the findings and their significance. This paper describes the clinical findings made at our Vision Assessment Clinic. We report the proportions of children with visual dysfunction due to cortical pathology, and describe the range of apparent cognitive disorders encountered. The opportunity has yet to be taken for a more in-depth evaluation of the presumed specific cognitive visual deficits which we have identified.

\section{PATIENTS AND METHODS}

The Vision Assessment Clinic commenced in September 1992, and is held approximately twice a month. During the ensuing 30 months, 130 patients have been seen. Children are referred to this Clinic for the following reasons:

1. Estimation of visual function is difficult owing to communication problems:

(a) Infants and young children deemed by referring clinicians to have poor vision.

(b) Multiply handicapped children.

2. Apparently paradoxical visual behaviour for which an explanation and strategy of management is requested. 
Table I. Indications for referral to the Vision Assessment Clinic

\begin{tabular}{lc}
\hline Site of primary pathology & $\begin{array}{c}\text { No. of } \\
\text { cases }\end{array}$ \\
\hline Eye disorders (e.g. ROP, albinism) & 26 \\
$\begin{array}{l}\text { Optic nerve/chiasm disorders (e.g. ONH } \\
\text { pituitary tumours) }\end{array}$ & 10 \\
$\begin{array}{l}\text { Eye movement disorders (oculomotor apraxia } \\
\text { diagnosed) }\end{array}$ & 4 \\
Brain disorders & 90 \\
\cline { 2 - 2 } & 130 \\
\hline
\end{tabular}

ROP, retinopathy of prematurity; ONH, optic nerve hypoplasia.

All children were assessed by a Consultant Developmental Paediatrician, who summarised the relevant background data, and made a clinical assessment of the child's intellectual ability. The severity and nature of any motor disorder was also recorded.

The visual acuity was estimated using a range of different methods appropriate for each child. A minority of children were able to perform a standard Snellen visual acuity test. The majority, however, underwent assessment of acuity by means of preferential looking (Cardiff acuity test and/or Keeler acuity cards) and visual evoked potentials, according to protocols previously described. ${ }^{5}$ In addition, a range of other methods of assessment of visual function was employed, including optokinetic nystagmus, fixation and following, near reaching thresholds and awareness of faces. The functional binocular acuity was determined on the basis of the results of successfully completed tests appropriate for each child. Values are expressed as Snellen equivalents, in order to provide an easily understood index of visual resolution for both parents and teachers.

Visual fields were assessed, particularly for evidence of homonymous hemianopia, in all children in whom behavioural or confrontation methods could be used. A wide range of other tests of visual function were available and employed when indicated.

Table I illustrates the numbers of children who have been assessed and the distribution of sites of

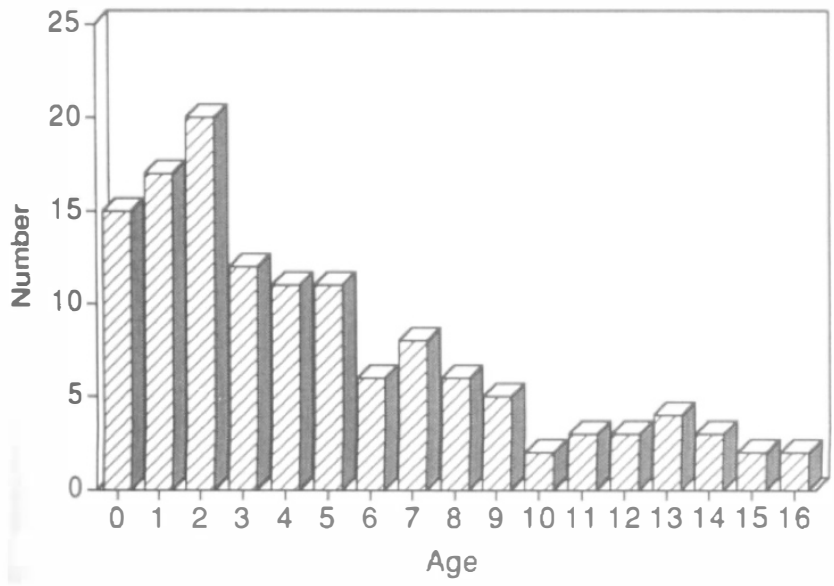

Fig. 1. The age distribution of the population studied. visual dysfunction. Fig. 1 illustrates the age distribution of this population.

This report primarily concerns 90 children with brain pathology, and describes the spectrum of clinical features which these children manifest. Clinical assessment of each child included between 30 and 60 minutes of history taking. An open questioning strategy was initially employed, in order to ascertain the level of visual functioning and to elicit the specific problems encountered by parents and carers. This was followed by a structured questioning strategy, in which the following information was sought, depending upon the degree of impairment in vision. Questions concerning the presence or absence of 'blind-sight' were asked for the most profoundly impaired children. For children with severe visual impairment due to brain damage, questions were asked concerning eye contact, the distance at which a smile might be returned, social interaction and visual behaviour. For children with a visual acuity of $6 / 60$ or better, questions were asked eliciting evidence of cognitive visual dysfunction in each of the five domains described below.

\section{RESULTS}

A total of 90 of the 130 patients referred to this Clinic showed evidence of visual dysfunction due to cortical visual impairment. These children manifested a spectrum of severity of impairment in vision, ranging from complete blindness, through severe impairment in which only rudimentary visual function was detected and visual impairment with field loss and/ or agnostic deficits, to reduced acuity only, with no apparent agnostic defects. Table II illustrates the proportions of cases in each group.

\section{Cortical Blindness}

Sixteen children showed no evidence of preferential looking behaviour and little, if any, evidence of vision as measured by visual evoked potentials. All showed moderate or severe intellectual impairment and all but one (who had diplegia) were quadriplegic or quadriparetic. Only 2 were mobile, and both these children showed evidence of navigational blind-sight, avoiding obstacles in their path if these were to the side but not if they were directly in front. These 2 children had initially shown no evidence of vision during the first 2 years of life, but subsequently became able to navigate and locate peripheral large, moving (but not static) targets.

Table II. Categories of cortical visual impairment $(n=90)$

\begin{tabular}{ll}
\hline Cortical blindness & $18 \%$ \\
Severe visual impairment & $10 \%$ \\
Visual impairment with field loss and/or & $52 \%$ \\
agnostic defects & \\
$\begin{array}{l}\text { Reduced acuity with no obvious agnostic } \\
\text { defects }\end{array}$ & $20 \%$ \\
\hline
\end{tabular}


A severe hypoxic ischaemic insult during the first 12 months of life was the cause of brain damage in 12 cases. Posterior hydrocephalic damage was the primary cause in 2 patients, while trauma and agenesis of the corpus callosum accounted for the remaining 2 .

\section{Cortical Visual Impairment with Severe Brain Damage}

There were 9 children with cortical visual impairment for whom it was possible to obtain an estimate of binocular visual acuity (primarily by means of visual evoked potentials) but whose generalised severe brain disturbance precluded any estimation of cognitive visual function. The spectrum of causation of pathology in this group was similar to that for the cortically blind group.

\section{Cognitive Visual Dysfunction}

Twenty children showed paradoxes in visual behaviour which parents and other carers found difficult to understand. Eight of these children had left homonymous visual field impairment (out of a total of $23(34 \%)$ ), and 3 had right visual field impairment (out of a total of $16(18 \%)$ ). One child (case 60) exhibited consistent bilateral lower visual field impairment. The remaining 8 showed no gross visual field loss. All but 1 of the 20 children were over the age of 4 years. The average age was 8.4 years.

The disorders of cognitive visual function which were described, and which were observed in children, could be divided into five categories, and involved impairment of:
1. Recognition.
2. Orientation.
3. Depth perception.
4. Perception of movement.
5. Simultaneous perception.

Fig. 2 illustrates the case numbers of the children who showed evidence of these disorders and also shows the combinations of disorders which these children were found to have. The presence and side of hemianopic field defects are also illustrated. Fig. 3 shows the range of visual acuities measured for these children.

\section{Impaired Recognition}

Impaired recognition was the commonest problem and took many forms and varied in degree. A clearcut classification into the forms of visual agnosia seen in adulthood could not easily be applied, owing to difficulties in knowing the normal range of cognitive perception for each age, compounded by the

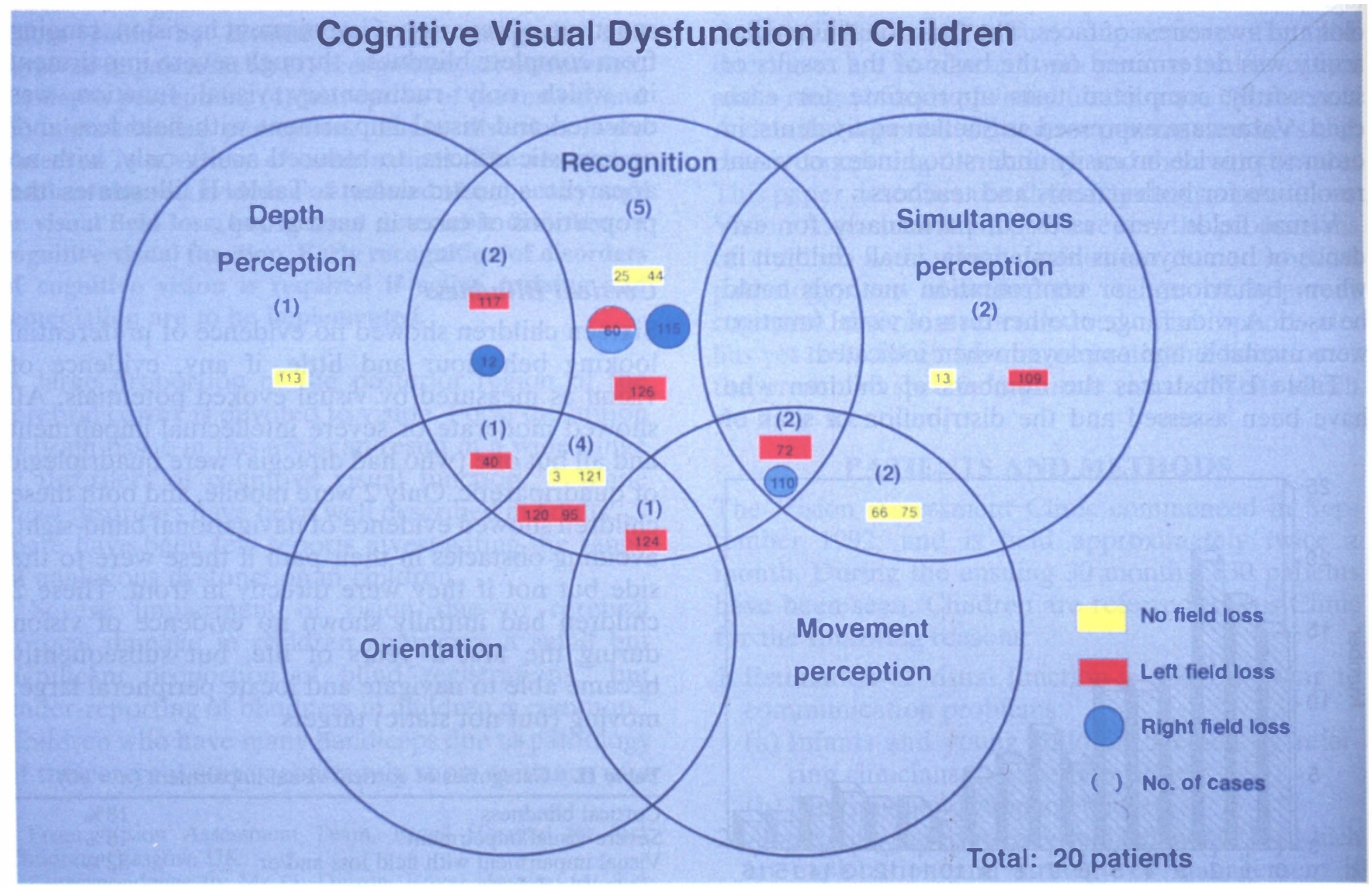

Fig. 2. Venn diagram, illustrating the range of cognitive disorders in visual function and the numbers of children affected by these disorders. The case numbers are included for cross-reference with the text. 


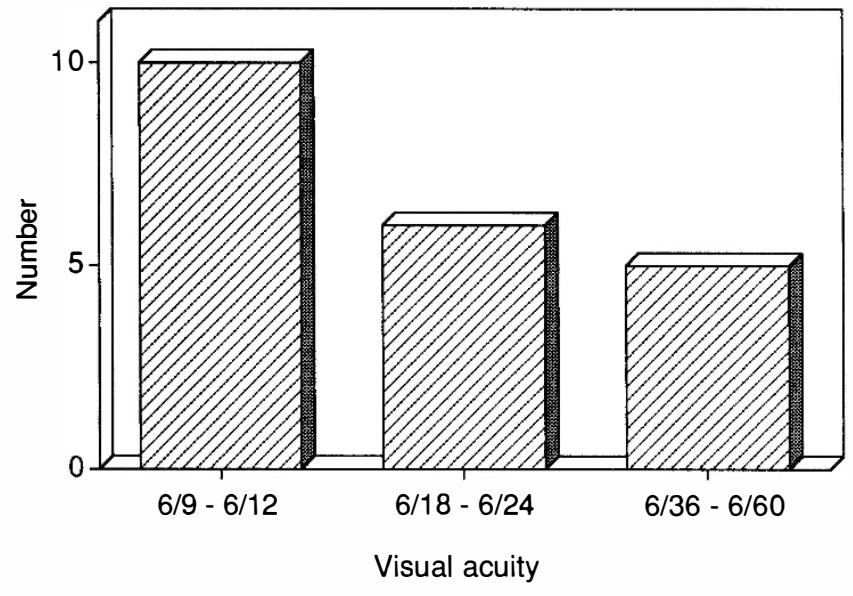

Fig. 3. The visual acuities of the patients shown in Fig. 2.

associated reduced visual acuity and impaired cognition. However, obvious inconsistencies in visual behaviour were the hallmark of these disorders.

Difficulties in recognising people comprised the most common disorder (15 cases). In the majority of cases $(95,110,115,117,120,121,124$ and 126), the description given was one of being unable to

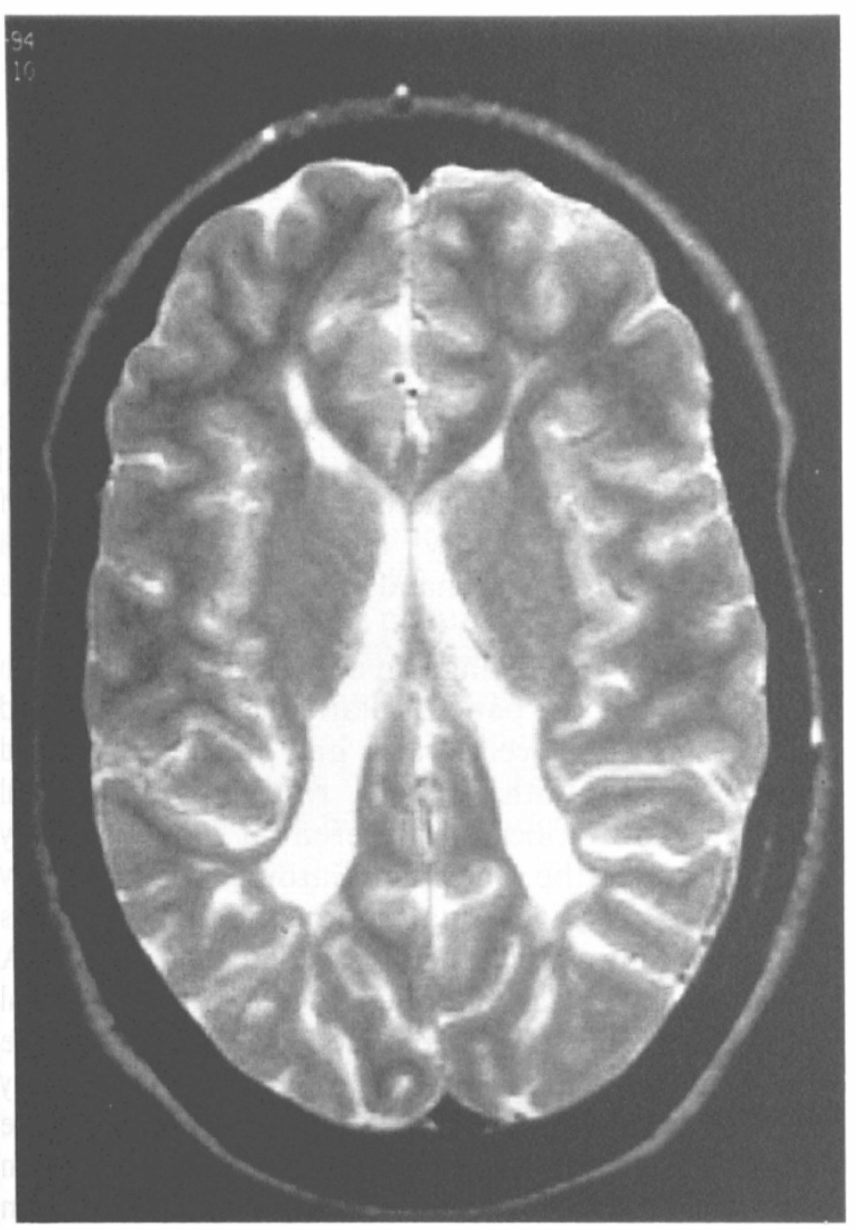

Fig. 4. Case 126. T2-weighted MRI axial section of the brain, showing extensive white matter loss in both posterior parietal and occipital lobes, causing a scalloped contour to the occipital horns of the lateral ventricles. recognise well-known individuals when they were in a group, but not apparently having the same difficulty on a one-to-one basis. Other individual descriptions included the following:

A complete inability to recognise people (case 3 ).

An ability to recognise first-degree relatives only (case 12).

An inability to recognise people from photographs, but not in real life (case 72).

A difficulty in discriminating unknown individuals from known, but not vice versa (case 40).

Visual field testing revealed evidence of left homonymous hemianopia which was incomplete in 3 cases, and complete in 4 others. Right visual field loss was seen in 2 patients, 1 of whom was manifestly left-handed. Bilateral lower field loss was present in 1 child. No gross visual field loss was elicited in the remaining 4 patients. Two children (cases 121 and 126) were investigated in more detail by Professor Andrew W. Young (Cambridge University, Department of Psychology), who found in both cases that there was a significant disability in identifying and naming a range of objects seen in standardised

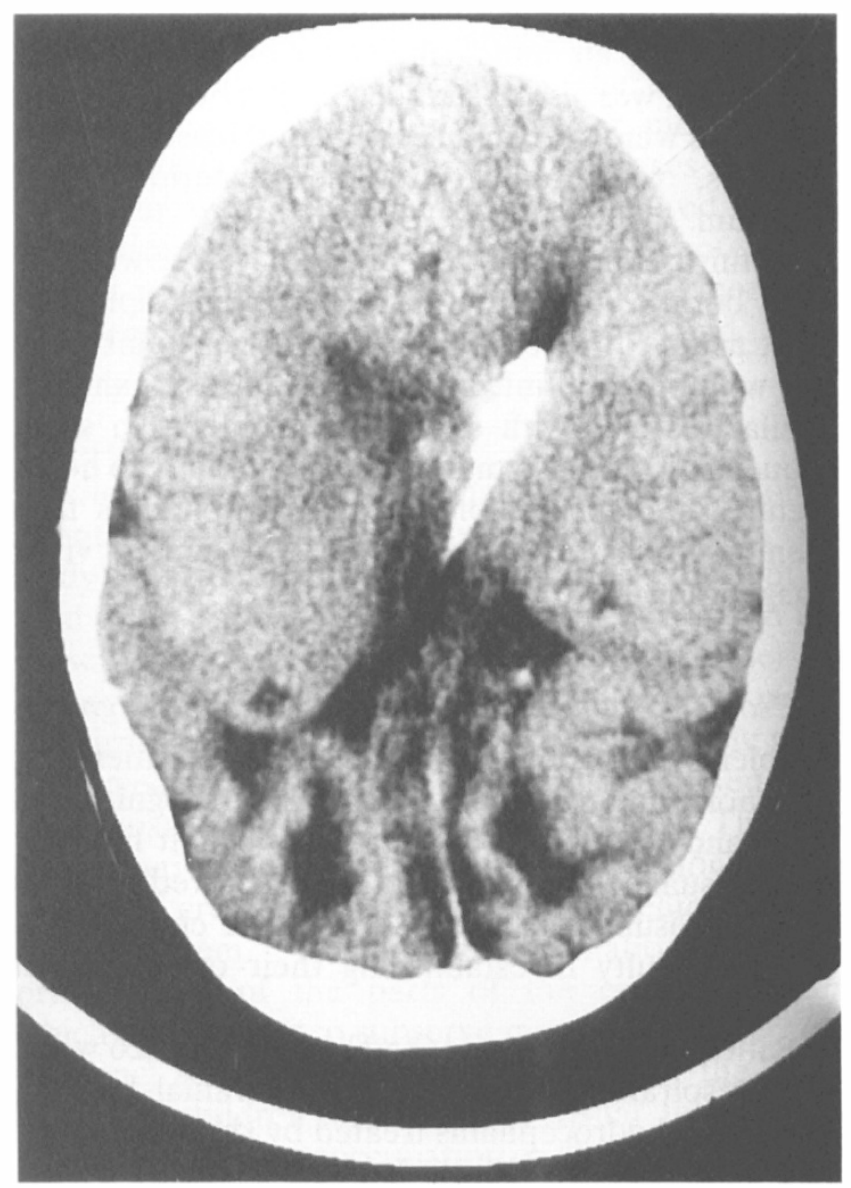

Fig. 5. Case 120. Axial CT section of the brain showing old bilateral occipital infarction following acute hydrocephalus at 8 months. There is a ventricular catheter in situ. 
photographs (designed for testing adults). He came to the conclusion that the children he saw do not conform to the pattern of visual agnosia established for adults, and for patient 126 (who is normal both intellectually and socially) he concluded that a form of integrative agnosia, in which the component parts of the images presented failed to add up to a coherent whole, may have contributed to the deficiency. Fig. 4 illustrates the CT scan for case 126.

Alexia without agraphia was seen in one 8-year-old child (case 3), who had developed hydrocephalus secondary to Haemophilus meningitis at the age of 2 years. Despite having a visual acuity of $6 / 9$, this child, who had bilateral extensive occipital damage associated with damage to the posterior corpus callosum, had developed severe cognitive visual dysfunction. This comprised an inability to read out loud, but a rudimentary appreciation of the syntactical meaning of text, combined with an intact ability to write well! She had a complete inability to recognise faces (prosopagnosia) and a degree of impaired orientation (see below), characterised by both an inability to recognise anyone by visual means and great difficulty in finding her way around in new environments, combined with a tendency to lose everyday items. A form of anomia featuring an inability to name pictures, but an intact ability to name all objects when held, was also described. Similar but less severe problems were seen in a second child (case 25), aged 4 years, with damage to the posterior corpus callosum.

A third child (case 60), aged 15 years, who had been born pre-term and had asymmetric diplegia, a right acuity of $6 / 18$ and a left divergent amblyopic eye with bilateral inferior visual field loss, exhibited similar features with associated problems in shape sequencing. She also manifested an inability to hold a picture in her mind or to construct pictures from memory in association with a very limited visual imagination.

\section{Problems with Orientation (Topographic Agnosia)}

Problems with orientation were seen in 6 patients, all of whom had variants of impaired recognition of faces and 4 of whom had evidence of left homonymous visual field impairment. None showed evidence of right visual field loss. All of these children had great difficulty in establishing their orientation in visual space.

Patient 40 (age 7 years) had been born at 26 weeks with respiratory distress, and intracranial bleeding had led to hydrocephalus treated by shunting (which had to be resited 3 months later). He showed normal social interaction and intellectual development. His preferential looking acuity (Cardiff test) was $6 / 12$ for each eye with a left homonymous hemianopia. He has difficulty in identifying both simple shapes and people's faces and, until recently, when a range of educational strategies were introduced, he was unable to find his way around in his newly acquired motorised wheelchair, both in the home and outside.

Patients 3, 95, 121 and 124 were all described as having a tendency to get lost easily, particularly in new environments, but in patient 120 (age 5 years) the problem is particularly profound. Intra-uterine intraventricular haemorrhage, due to allo-immune thrombocytopenic purpura, had led to hydrocephalus which required shunting at 8 months. CT scan images initially showed marked expansion of the lateral ventricles, particularly posteriorly. The later appearances are shown in Fig. 5. He has a degree of optic atrophy and nystagmus with near reduced Snellen visual acuities of 6/12 right and 6/18 left, and a distance binocular Snellen acuity of $6 / 36$ and a left hemianopia. He walks independently with mild diplegia and shows normal social interaction. $\mathrm{He}$ has difficulty recognising his mother and father when they are in the presence of others, but in particular 'he gets lost if he turns a corner', even within his own home.

\section{Problems with Depth Perception}

Problems with depth perception were apparent in an early form in a $2^{1 / 2}$-year-old girl (case 113) who had sustained a hypoxic ischaemic episode as a sequel to near cot death. MRI scanning at the time revealed haemorrhagic infarction of the right occipital lobe. Despite a binocular preferential looking visual acuity of $6 / 24$, this child displayed a complete inability to locate and reach for objects in three-dimensional space. To compensate, she had adopted a strategy of reaching beyond every target and of gradual approximation of her hand, in order to grasp the object of interest.

An inability to distinguish a line on the floor from steps was seen in 2 patients. Patient 12 was examined at the age of 11 years. She had sustained a head injury at 15 months of age, resulting in cerebral atrophy. The binocular preferential looking acuity was $6 / 12$, but the Sheridan-Gardiner visual acuity was 6/60. A right inattention hemianopia was present, along with difficulty recognising people. A mild diplegia was manifest with normal social interaction, but with reduced intelligence. The principal problem, however, was a complete inability to discriminate a step from a line on floor, and vice versa. A similar symptom complex was seen in patient 117 (aged 7 years), who had required an emergency caesarian section for fetal distress. He has a normal intellect, but mild spastic diplegia. Despite a visual acuity of 6/12 (and a left homonymous hemianopia), this child is unable to distinguish steps from lines and also had difficulty recognising 
people. Impaired horizontal pursuit eye movements are associated with compensatory convergence. ${ }^{6}$

\section{Impaired Movement Perception}

Impaired movement perception was seen in 5 children. In 4 cases $(66,72,75$ and 110$)$ this occurred in association with a degree of impaired simultaneous perception, and there was concomitant impaired recognition of people in 2 of these cases (72 and 110). The condition was not seen in isolation.

Patient 66 (aged 9 years) was born prematurely. Recent MRI scanning has shown peri-ventricular leucomalacia (PVL) with dilated ventricles anteriorly, indicative of a pre-natal event at about 26 weeks. She has spastic quadriplegia and sits with support, but has only moderate intellectual impairment. She has a convergent squint with a visual acuity of $6 / 36$ (Kay pictures) in the fixing eye. Her mother volunteered a spontaneous account of her visual problems at the initial consultation. During car travel she only sees distant targets but not near ones. Dogs and cats are seen only when they move slowly, and she gives a consistent and accurate count of the rabbits on her lawn, but omits to count the moving ones. She is able to count fingers on a hand which is moving very slowly, but is unable to do so when it moves more quickly. These observations are consistent with a degree of akinetopsia (an inability to see movement). When she describes pictures, she will only see one person a time in a family photograph. When shown a picture of a person with a hat with a flower in it, she immediately identified the flower, but was unaware of the presence of the person until it was drawn to her attention. Similar responses were obtained when she was shown a number of other simple pictures. This phenomenon is consistent with a form of simultanagnosia and was observed when she viewed many other pictures in which the content was commensurate with a $6 / 36$ visual acuity.

Very similar descriptions of impaired (but not absent) ability to see movement were given for 3 other patients (cases 72, 75 and 110), who all showed clinical evidence of bilateral cortical dysfunction.

\section{Impaired Simultaneous Perception}

Impaired simultaneous perception was described in isolation in 2 children (cases 13 and 109). In patient 13 (aged 12 years), neonatal asphyxia had resulted in spastic quadriplegia. $\mathrm{He}$ is able to sit with support and has remarkably normal intellect and social interactions. The binocular Snellen visual acuity was $6 / 24$. He is able to see movement, but when shown pictures he will only describe one component until another is drawn to his attention. He has learned to read, but primarily on account of the efforts of his parents, who have empirically discov- ered that masking all the text, bar the word of interest, is required.

A similar description was given for patient 109 (aged 9 years), in whom bilateral severe shunted hydrocephalus with a large porencephalic cyst was the cause of apparent initial cortical blindness; but the subsequent development of $6 / 60$ corrected binocular visual acuity, with a left incomplete homonymous hemianopia and left convergent squint, were observed. This child, again, is able to describe only the component parts of a picture (e.g. a clock in which he will describe the hands but not the whole clock), and will describe other parts of the picture only when they are drawn to his attention.

It is perhaps not surprising that no descriptions of disorders of cognitive visual function were given for any children with visual acuities of less than $6 / 60$. At lower visual acuities, image degradation no doubt impairs all aspects of visual behaviour and makes inconsistencies in such behaviour less likely to be apparent or manifest.

\section{DISCUSSION}

This is a retrospective clinical observational study, in which we were progressively learning more from our first cohort of patients. A prospective questioning strategy is now being devised on the basis of the observations we have described. For most of the patients described, the apparent paradoxes in visual behaviour were spontaneously volunteered by the parents/carers. Such an approach is potentially subject to a degree of bias, and this paper needs to be interpreted with such reservations in mind.

\section{Cortical Visual Impairment}

It is important to recognise that a large proportion of infants with cortical visual impairment $(65 \%$ in one study $)^{7}$ may ultimately develop vision sufficient to read print. Similarly, the population described in the present study is not static and the deficits observed are evolving. Children with severe cortical visual impairment may go on to develop remarkably wellpreserved perception of movement, combined with an almost paradoxical navigational vision. This has been ascribed to the possible preservation of the temporal crescent, and to the extrageniculostriate visual system. ${ }^{8}$ A further explanation may be preservation of the parts of the cerebral cortex which are thought to subserve movement perception in man. ${ }^{3}$ A feature common to many of the more impaired children was variable visual function, in which tiredness, preoccupation or distraction leads to an apparent reduction in visual acuity, whilst familiarity with the environment may lead to an apparently better acuity. These features have been well described previously. ${ }^{9}$ 


\section{Cognitive Visual Impairment}

The information elicited from the children and parents concerning complex anomalies of visual behaviour was obtained both from the history and from observation of the child's visual behaviour during a 1-2 hour consultation and testing period. More detailed appraisal and in-depth study would, no doubt, reveal a wider range of deficits and provide a more rigorous categorisation of their nature.

Poor acuity may be the primary cause of apparent cognitive visual function in some cases, but the paradoxical visual problems observed in the visual behaviour of the 20 children we saw with inconsistent visual function, make such an explanation unlikely.

Focal visual cognitive dysfunction in adults resulting from focal trauma or ischaemia leads to a detectable loss of a previously intact cognitive visual function (e.g. face recognition). By contrast, the developmental plasticity of children and the fact that functional impairment is associated with impaired development and not loss of a previously acquired function, conspire to produce a complex picture of cognitive visual disturbance which may well respond to training and gradually become less evident. We believe, however, that there is a need to develop a classification of paediatric visual agnosias. This will provide a greater understanding of the child's visual problem, the destined role for different brain territories and the adaptive developmental plasticity of intact tissue.

A considerable amount of brain tissue is devoted to analysis and interpretation of the visual world, and to visual memory and imagination; these are required for planning tasks and for orientation, both on a small scale and on a large scale (e.g. route finding). Considering the extent of pathology observed in many of the children we have examined, it is remarkable that agnostic deficits are not more commonly identified.

\section{Recognition of Faces}

Recognition of faces is a highly complex task which entails a process of rapid pattern matching between the image seen, and memory banks of stored facial imagery, followed by the cognitive process of making this connection in order for recognition to take place. The 2 children in our study who were investigated in more detail for their impaired recognition, were found to be unable to recognise a wide range of objects as well as faces, and thus had problems in integrating the data into a meaningful concept. In both hydrocephalus and periventricular leucomalacia the white matter pathways, linking adjoining cortical territories which subserve different cognitive functions, are deficient, and it is not surprising that such children have complex disorders of the recognition process.
A recent study of face recognition in children with early brain damage has shown a range of face recognition disorders, including disability in recognising the linguistic and emotional components of facial expression. ${ }^{10}$

In many cases, particularly in those with additional topographic agnosia, there was a left homonymous hemianopia and other evidence of right occipital dysfunction. This is consistent with observations in adults, in whom right brain dysfunction is more commonly associated with these phenomena. ${ }^{2,3}$

The question is whether it is possible in children with recognition problems to circumvent their disabilities by specifically designed education, training or appropriate strategies. One child in our series carries a pendant which he gives to his teacher to wear, so that he is able to recognise her.

\section{Impaired Orientation}

Impaired orientation was common and occurs both on a large scale (getting lost in new environments) and on a small scale (remembering where one has put things). In 3 of our patients, the parents have reported that by colour-coding doors in the house, and by constantly drawing attention to visual and other orientational cues (e.g. wind and sun direction), the tendency for the children to get lost has been significantly reduced. Training in putting things 'in their place' also helps the small-scale problem. A novel, more structured approach to the development of such educational strategies is warranted.

\section{Impaired Depth Perception}

Impaired depth perception in our patients appeared to have a deeper cognitive basis than just impaired stereopsis, in which coarse appreciation of depth is not impaired. The cortical appreciation of depth has a complex cellular basis. ${ }^{11}$ The interpretation of where things are in visual space, with respect to oneself, is very complex and prone to disturbance. We suggested a strategy of training in using the feet to explore the world beyond the 'line/step' in the floor, which appears to have proved helpful.

\section{Impaired Perception of Movement}

Impaired perception of movement was first described in an adult patient with bilateral pathology at the junctions of the parietal temporal and occipital lobes. $^{12}$ A number of cases have been reported since and the subject has been fully reviewed..$^{13}$ It is, therefore, not surprising that children with pathology in the same area should manifest behaviour consistent with either a disability or an inability to see movement (akinetopsia).

\section{Impaired Simultaneous Perception}

Impaired simultaneous perception is seen in adults 
with bilateral occipitoparietal dysfunction. ${ }^{2,3}$ Behaviour consistent with this dysfunction was seen in those children with such bilateral pathology. It is important to recognise the possible presence of simultanagnosia, as this fundamentally affects training and education, in which the presentation of sequential single, simple images is the logical means of optimising transfer of information.

Fig. 2 shows the associations between the different cognitive disorders we observed. This diagram is equally notable for its lack of associations. For example, akinetopsia was not associated with impaired depth perception. The current state of knowledge concerning cognitive visual disorders in adults is consistent with the patterns of association we have found.

In conclusion, cortical visual impairment in children appears to range from cortical blindness to a reduction in acuity, but there is a significant group in whom it is dysfunction of the centres required for analysis and interpretation of the visual world which constitutes the principal visual problem. Training may well improve such visual dysfunction, ${ }^{14}$ and there is a need to develop appropriate educational protocols which cater for the needs of these children. Early intervention and training in infants and young children has been shown to enhance visual function significantly. ${ }^{15}$ Anticipation of the potential for disorders of cognitive vision and appropriate intervention could prove effective in minimising the problems associated with these disorders.

The following grant-awarding bodies have contributed to the work described in this paper: The Wellcome Trust, the Scottish Office Home and Health Department, and the WRVS.

Key words: Cortical blindness, Prosopagnosia, Akinetopsia, Topographic agnosia, Simultanagnosia, Astereocognosis.

\section{REFERENCES}

1. Foley J. Central visual disturbances. Dev Med Child Neurol 1987;29:116-20.
2. Grusser O-J, Landis T. Visual agnosias and other disturbances of perception and cognition. Vision and visual dysfunction, vol 12 (Cronly Dillon JR, general editor). Basingstoke, Hampshire: Macmillan Press, 1991.

3. Dutton GN. Cognitive visual dysfunction. $\mathrm{Br} \mathrm{J}$ Ophthalmol 1994;78:723-6.

4. Goggin M, O'Keefe M. Childhood blindness in the Republic of Ireland: a national survey. Br J Ophthalmol 1991;75:425-9.

5. Mackie RT, McCulloch DL, Saunders KJ, Ballantyne J, Bradnam MS, Day RE, Dutton GN. Comparison of visual assessment tests in multiply handicapped children. Eye 1995;9:136-41.

6. Beigi B, O'Keefe M, Logan P, Eustace P. Convergence substitution for paralysed horizontal gaze. $\mathrm{Br} \mathrm{J}$ Ophthalmol 1995;79:229-32.

7. Jan JE, Wong PKH, Groenveld M, Flodmark O, Hoyt CS. Travel vision 'Collicular visual system?' Pediatr Neurol 1986;2:359-62.

8. Jan JE. Neurological causes and investigations. In: Fielder AR, Best AB, Bax MCO, editors. The management of visual impairment in childhood. Clinics in developmental medicine 128. London: MacKeith Press, 1993:48-61.

9. Jan JE, Greenwold M, Sykarda AM, Hoyt CS. Behavioural characteristic of children with permanent cortical visual impairment. Dev Med Child Neurol 1987;29:571-6.

10. Mancini J, de Schonen S, Deruelle C, Massoulier A. Face recognition in children with early right or left brain damage. Dev Med Child Neurol 1994;36:156-66.

11. Zeki S. Further unresolved problems of integration. The representation of space. In: A vision of the brain. Oxford: Blackwell Scientific, 1993: 337-42.

12. Zihl J, Cramon D von, Mai N. Selective disturbance of movement vision after bilateral brain damage. Brain 1983;106:313-40.

13. Zeki S. Cerebral akinetopsia (cerebral visual motion blindness). Brain 1991;114:811-24.

14. Merrill MK, Kewman DG. Training of color and form identification in cortical blindness: a case study. Arch Phys Med Rehabil 1986;67:479-83.

15. Sonksen PM, Petric A, Drew KJ. Promotion of visual development of severely visually impaired babies: evaluation of a developmentally based programme. Dev Med Child Neurol 1991;33:320-35. 